\title{
Variational Approximations for Intersite Soliton in a Ablowitz-Ladik-Cubic Discrete Nonlinear Schro̊dinger Equation
}

\section{Azzahro Fitri Azadi, Mahdhivan Syafwan, Admi Nazra, Riza Azfa, and Zita Putri Netris}

Department of Mathematics, Andalas University, Limau Manis, Padang, Indonesia, 25163

\section{Abstract}

This paper investigates the existence of intersite soliton in the Ablowitz-Ladik-cubic discrete nonlinear Schrödinger (AL-cubic DNLS) equation in the anti-continuum limit by using a variational approximation (VA) method. The AL-cubic equation interpolates the integrable Ablowitz-Ladik DNLS equation and the non-integrable cubic DNLS equation. We obtain that the approximated solitons are in good agreement with those resulted from numerics. We also show that the approximated solitons are valid for small coupling

Corresponding Author: Mahdhivan Syafwan mahdhivan@sci.unand.ac.id

Received: 19 February 2019 Accepted: 5 March 2019 Published: 16 April 2019 Publishing services provided by Knowledge E

(c) Azzahro Fitri Azadi et al. This article is distributed under the terms of the Creative Commons Attribution License, which permits unrestricted use and redistribution provided that the original author and source are credited.

Selection and Peer-review unde the responsibility of the ICBSA Conference Committee.

\section{Introduction}

One of equations that is often studied in both the theory aspect and in the context of its application is the discrete nonlinear Schrödinger (DNLS) equation. This is because the equation models many important phenomena, such as an array of nonlinear optical waveguides etched onto a semiconductor material (AIGaAs) [1], matter wave dynamics in Bose-Einstein condensates trapped in optical lattices and molecular biology (modeling the DNA double strand) [2].

The most interesting feature about the DNLS equation is the existence of soliton. Soliton is a localized solution that has properties: it maintains its shape and propagates at a constant speed even after collision [3]. In the context of its application, Tagg [4] described the use of solitons in fiber optic communication systems which provide highly accurate signal transmission over extremely long distance. This is very important in the development of future communication technology.

The general form of the DNLS equation is given by [2]

$$
i \dot{\varphi}_{n}=\varepsilon\left(\varphi_{n+1}-2 \varphi_{n}+\varphi_{n-1}\right)+F\left(\varphi_{n+1}, \varphi_{n}, \varphi_{n-1}\right)
$$


where $\varphi_{n} \equiv \varphi_{n}(t) \in \mathbb{C}$ is a wave function at time $t \in \mathbb{R}^{+}$and site $n \in \mathbb{Z}$, $\dot{\varphi}_{n}$ represents the derivative of the function $\varphi_{n}$ with respect to $t, \varepsilon>0$ represents coupling constant and $F$ is a nonlinear term that has several forms:

1. Ablowitz-Ladik (AL)

$$
F_{A L}=\frac{1}{2}\left|\varphi_{n}\right|^{2}\left(\varphi_{n+1}+\varphi_{n-1}\right)
$$

2. Cubic

$$
F_{\text {cub }}=\left|\varphi_{n}\right|^{2} \varphi_{n} .
$$

In 1975-1976, Ablowitz and Ladik [5] showed that the DNLS equation with nonlinear term (2) is integrable, while equation (3) is not integrable. For non-integrable equations, an analytic approach is needed to approximate the solution. One of the methods which is well known and has been long used to approximate solutions (including the localized states) of a nonlinear evolution equation is the so-called variational approximation (VA). Formulation of this method is based on theory of Lagrangian and Hamiltonian mechanics (see, e.g., [6]). The success of this method depends heavily on the trial function (ansatz) used in approaching the desired solution.

VA methods have been used in various equations, including in determining soliton solution in the cubic DNLS equation (3). Aceves et al [7] used the VA method to approximate the onsite soliton solution (i.e centred on a lattice site). In addition, VA has been also applied to approximate the intersite soliton solution (i.e centred between two adjacent lattice sites) with symmetrical configuration by Cuevas et al [8]. Furthermore, Kaup et al [9] developed the VA formulation to approximate the asymmetric intersite soliton solution. The ansatz function used in [8] and [9] applies for the case $\varepsilon \approx 0$ or known as the anti-continuum limit.

The results of VA have been confirmed its validation through numerical comparisons for certain parameter values. To justify rigorous VA validation, Chong et al. [10] have developed a theorem that can be used as a tool of validation of the VA results. Chong et al then confirmed that a trial function for solitons with more parameters provides a more accurate approximation.

In this paper, the VA method will be applied to determine intersite soliton of the following equation:

$$
i \dot{\varphi}_{n}=\varepsilon\left(\varphi_{n+1}-2 \varphi_{n}+\varphi_{n-1}\right)+\frac{\alpha}{2}\left|\varphi_{n}\right|^{2}\left(\varphi_{n+1}+\varphi_{n-1}\right)+(1-\alpha)\left|\varphi_{n}\right|^{2} \varphi_{n} .
$$

Equation (4) can be viewed as an interpolation of the Ablowitz-Ladik DNLS equation (when $\alpha=1$ ) and the cubic DNLS equation (when $\alpha=0$ ). Equation (4) is then called the Ablowitz-Ladik-cubic discrete nonlinear Schrödinger (AL-cubic DNLS) equation. 


\section{The Formulation of Variational Approximation}

In this section we describe the formulation of variational approximation method. This is referred from reference [11]. Let $u(x, t)$ with $t \geq 0$ and for every $x \in \Omega \subseteq \mathbb{R}$, satisfy partial differential equations in the form

$$
u_{t}=f_{v}\left(x, u, u_{x}, u_{x x}\right)+f_{n v}\left(x, u, u_{x}, u_{x x}\right)
$$

where $f_{v}$ denotes a variational term and $f_{n v}$ denotes a nonvariational term, i.e there is a function $F\left(x, u, u_{x}\right)$ such that

$$
f_{v}\left(x, u, u_{x}, u_{x x}\right)=\frac{\partial F}{\partial u}-\frac{d}{d x} \frac{\partial F}{\partial u_{x}} .
$$

In the variational case (that is by assuming that $f_{n v} \equiv 0$ ), the stationary solutions of (6) are indeed extrema of the functional (called Lagrangian)

$$
L:=\int_{\Omega}-F\left(x, u, u_{x}\right) d x
$$

Suppose a variational solution can be written in the form $u=U\left(x, A_{1}, \ldots, A_{n}\right)$ with a finite number of parameters $A_{1}, A_{2}, \ldots, A_{n}$. The result of integration (7) using such a variational solution is called effective Lagrangian $\left(L_{\text {eff }}\right)$. Thus the extreme value for effective Lagrangian satisfies

$$
\frac{\partial L_{\mathrm{eff}}}{\partial A_{i}}=\int_{\Omega} \frac{\partial U}{\partial A_{i}}\left[\frac{d}{d x} \frac{\partial F}{\partial U_{x}}-\frac{\partial F}{\partial U}\right] d x, \quad i=1,2, \ldots, n .
$$

Suppose now that the parameters are time-dependent functions, denoted as $A_{i}(t)$. Thus from equation (5) we obtain the following relationship

$$
\frac{\partial F}{\partial u}-\frac{d}{d x} \frac{\partial F}{\partial u_{x}}=\sum_{j=1}^{n} \frac{\partial U}{\partial A_{j}} \dot{A}_{j}-f_{n v}\left(x, u, u_{x}, u_{x x}\right),
$$

where $\dot{A}_{j}=\frac{d A_{j}}{d t}$. Upon substituting equation (9) into equation (8), then for every $i$ we obtain

$$
\frac{\partial L_{\mathrm{eff}}}{\partial A_{i}}=\int_{\Omega} \frac{\partial U}{\partial A_{i}}\left[f_{n v}-\sum_{j=1}^{n} \frac{\partial U}{\partial A_{j}} \dot{A}_{j}\right] d x .
$$

Suppose

$$
M_{i j}=\int_{\Omega} \frac{\partial U}{\partial A_{i}} \frac{\partial U}{\partial A_{j}} d x,
$$

then equation (10) for each $i=1,2, \ldots, n$ can be rewritten as follows

$$
\sum_{j=1}^{n} M_{i j} \dot{A}_{j}=-\frac{\partial L_{\mathrm{eff}}}{\partial A_{i}}+\int_{\Omega} \frac{\partial U}{\partial A_{i}} f_{n v} d x .
$$


For discrete system in space, equation (12) can be changed analogously to be

$$
\sum_{j=1}^{n} M_{i j} \dot{A}_{j}=-\frac{\partial L_{\mathrm{eff}}}{\partial A_{i}}+\sum_{m \in \Phi \subset \mathbb{Z}} \frac{\partial U_{m}}{\partial A_{i}} f_{n v} .
$$

In brief, the systematic steps of the VA method for both variational and nonvariational cases are given as follows:

1. Formulate the Lagrangian of the variational part of the governing equation.

2. Propose a reasonable trial function (ansatz) which contains a finite number of parameters (called variational parameters).

3. Substitute the proposed ansatz into the Lagrangian and evaluate the resulting sums (for discrete systems) or integrations (for continuous system).

4. Find the critical points of the variational parameters by solving the corresponding system (12) [for continuous system] or system (13) [for discrete system].

\section{Variational Approximation of AL-cubic DNLS Equation}

By performing the separation of variables, $\varphi_{n}(t)$ can be written in the form

$$
\varphi_{n}(t)=Q_{n} e^{-i t},
$$

where $Q_{n}$ is a time-independent function. Next, by substituting equation (14) into (4), we obtain the following stationary equation

$$
\varepsilon\left(Q_{n+1}-2 Q_{n}+Q_{n-1}\right)-Q_{n}+\frac{\alpha}{2}\left|Q_{n}\right|^{2}\left(Q_{n+1}+Q_{n-1}\right)+(1-\alpha)\left|Q_{n}\right|^{2} Q_{n}=0
$$

In general, solutions for $Q_{n}$ are complex valued. However, in this paper we are only consider the real-valued solution. Therefore, equation (15) can be simplified to

$$
\varepsilon\left(Q_{n+1}-2 Q_{n}+Q_{n-1}\right)-Q_{n}+\frac{\alpha}{2} Q_{n}^{2}\left(Q_{n+1}+Q_{n-1}\right)+(1-\alpha) Q_{n}^{3}=0 .
$$

Equation (16) can be written as follows:

$$
f_{v}+f_{n v}=0
$$

where

$$
f_{v}=\varepsilon\left(Q_{n+1}-2 Q_{n}+Q_{n-1}\right)-Q_{n}+(1-\alpha) Q_{n}{ }^{3},
$$

and

$$
f_{n v}=\frac{\alpha}{2} Q_{n}^{2}\left(Q_{n+1}+Q_{n-1}\right)
$$


Next, we are ready to apply the VA method. The first step is to determine the Lagrangian formula for the variational part $\left(f_{v}\right)$, which is given by

$$
L=\sum_{n=-\infty}^{\infty}-\left(-\varepsilon Q_{n-1} Q_{n}+\frac{1}{2}(1+2 \varepsilon) Q_{n}{ }^{2}-\frac{(1-\alpha)}{4} Q_{n}{ }^{4}\right) .
$$

The second step is to select the appropriate ansatz function. In this paper, we are interested to find the approximation of the intersite soliton. Thus, the following ansatz function can be selected:

$$
Q_{n}=\left\{\begin{array}{c}
A e^{\eta(n-1)}, \quad n \leq-1 \\
B, \quad n=0,1 \\
A e^{-\eta(n-2)}, \quad n \geq 2
\end{array}\right.
$$

where $A, B$ and $\eta$ are real valued variational parameters.

The third step is to substitute ansatz (21) into equation (20) and then evaluate the resulting sum. This gives the effective Lagrangian as follows

$$
\begin{aligned}
L_{\mathrm{eff}}=-\frac{1}{2} \frac{1}{e^{4 \eta}-1}( & B^{4}+4 \varepsilon A B-B^{4} \alpha-B^{4} e^{4 \eta}+2 B^{2} e^{4 \eta}+2 e^{2 \eta} A^{2}-2 B^{2}-2 \varepsilon B^{2} \\
& -4 \varepsilon A^{2} e^{3 \eta}+2 A^{2} e^{4 \eta}-A^{4} e^{4 \eta}-4 \varepsilon A B e^{4 \eta}+4 e^{2 \eta} A^{2} \varepsilon-4 A^{2} e^{\eta} \varepsilon \\
& \left.+B^{4} \alpha e^{4 \eta}+2 \varepsilon B^{2} e^{4 \eta}+A^{4} \alpha e^{4 \eta}+4 \varepsilon A^{2} e^{4 \eta}\right) .
\end{aligned}
$$

By substituting the effective Lagrangian (22), ansatz (21) and the nonvariational part (19) into equation (13), then the following system of equations is obtained

$$
\begin{gathered}
\mathscr{A}_{1}+\mathscr{A}_{2}=0, \\
\mathscr{B}_{1}=0, \\
\mathscr{C}_{1}+\mathscr{C}_{2}=0,
\end{gathered}
$$

where

$$
\begin{gathered}
\mathscr{A}_{1}=\frac{1}{e^{4 \eta}-1}\left(2 \varepsilon B+2 e^{2 \eta} A-4 \varepsilon A e^{3 \eta}+2 A e^{4 \eta}-2 A^{3} e^{4 \eta}-2 \varepsilon B e^{4 \eta}+4 A e^{2 \eta} \varepsilon\right) \\
\mathscr{A}_{2}=\frac{1}{e^{4 \eta}-1}\left(-4 A e^{\eta} \varepsilon+2 A^{3} \alpha e^{4 \eta}+4 A \varepsilon e^{4 \eta}+A^{3} \alpha e^{\eta}+A^{2} \alpha B e^{4 \eta}-A^{2} \alpha B+A^{3} \alpha e^{3 \eta}\right) \\
\mathscr{B}_{1}=-2 B^{3}+2 B-2 \varepsilon A+3 B^{3} \alpha+2 \varepsilon B+\alpha B^{2} A
\end{gathered}
$$




$$
\begin{gathered}
\mathscr{C}_{1}=-\frac{1}{\left(e^{4 \eta}-1\right)^{2}}\left\{e^{\eta} A^{2}\left(-2 \varepsilon-6 e^{2 \eta} \varepsilon+4 e^{\eta} \varepsilon+8 e^{3 \eta} \varepsilon-6 \varepsilon e^{4 \eta}+2 e^{\eta}+4 e^{3 \eta}\right)\right\} \\
\mathscr{C}_{2}=-\frac{1}{\left(e^{4 \eta}-1\right)^{2}}\left\{e^{\eta} A^{2}\left(-2 A^{2} e^{3 \eta}+2 e^{5 \eta}+2 A^{2} e^{3 \eta} \alpha-2 e^{6 \eta} \varepsilon+4 e^{5 \eta} \varepsilon+\alpha A^{2} e^{4 \eta}+e^{2 \eta} A^{2} \alpha\right)\right\}
\end{gathered}
$$

Due to complexity of the calculation, solutions for parameters $A, B$, and $\eta$ for given $\varepsilon$ and $\alpha$ in the above system can be determined numerically using the Newton-Raphson method.

\section{The VA Results and Comparisons with Numerics}

In this section, we compare the results of variational approximations with the corresponding numerical calculations. In this case, the numerical solution for the intersite soliton of (16) can be determined using the Newton-Raphson method where the VA solutions can be used as the initial guess. For illustrative example, in Figure 1 is shown a comparison between two intersite soliton solutions obtained from numerics and VA for coupling constant $\varepsilon=0.01 ; 0.02 ; 0.05$, and intepolation parameter $\alpha=0.01 ; 0.44$.

Fig. 1 also gives the solutions for varational parameters A, B, and $\eta$ which are obtained by solving the system of equations (23)-(25) numerically for given $\varepsilon$ and $\alpha$. From the figure we can observe that the VA soliton solutions and the numerical solutions have a very good agreement for some parameter values $\alpha$ and $\varepsilon$.

\section{Validation of VA Results}

Validation of VA results for discrete solitons in the stationary equation (16) is based on the justification formulated by Chong et al. [10]. To measure the accuracy of variational solution of equation (16), define its residual as

$$
R_{n}(Q)=\varepsilon\left(Q_{n+1}-2 Q_{n}+Q_{n-1}\right)-Q_{n}+\frac{\alpha}{2} Q_{n}{ }^{2}\left(Q_{n+1}+Q_{n-1}\right)+(1-\alpha) Q_{n}{ }^{3} .
$$

Note that if $Q$ is an exact solution, then $R_{n}(Q)$ will be zero for every $n$. Thus a variational solution will approach the exact solution if $R_{n}(Q) \rightarrow 0$ for every $n$.

Validation of the VA for the intersite soliton of the stationary AL-cubic DNLS equation (16) is given in the following propositions.

Proposition 1. Suppose $Q_{*}$ is a variational solution of intersite solitonof the stationary AL-cubic DNLS equation (16) which is expressed by ansatz (21), where the variational 

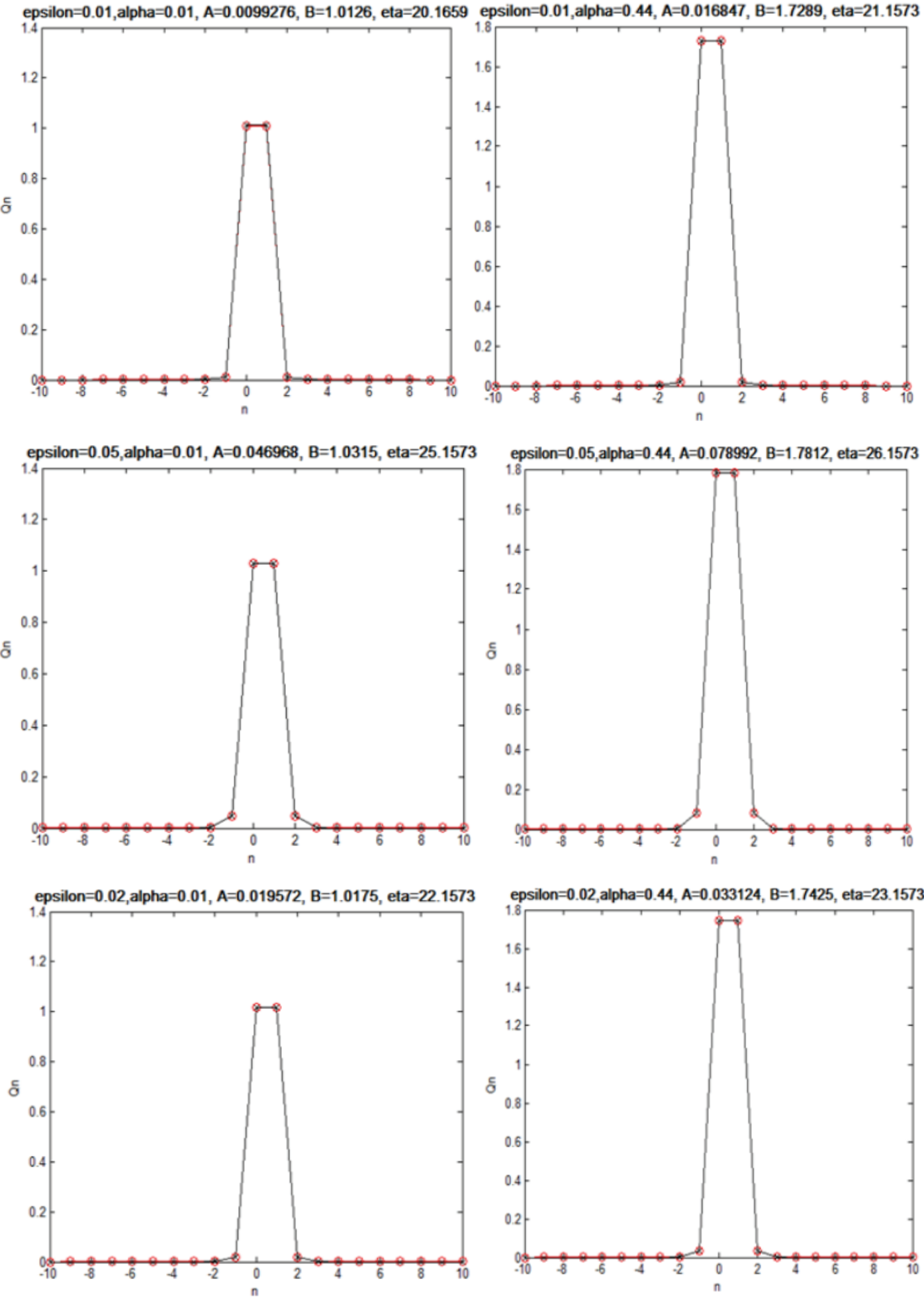

Figure 1: Comparison between numerical (circle markers) and variational (cross markers) solutions of the intersite soliton for some parameter values. 
parameters $\eta, A, B$ satisfy the equations (23), (24) and (25). Then there is $\varepsilon_{0}, \quad K>0$ such that for all $\varepsilon \in\left(0, \varepsilon_{0}\right)$, equation (16) has a unique solution $Q$ that satisfies

$$
\left\|Q-Q_{*}\right\|_{l_{2}} \leq K \varepsilon^{2} .
$$

Proof. Note that the rate of exponential decay of discrete soliton follows from the linear theory of difference equations. Therefore, the solution for parameter $\eta$ can be obtained by substituting $Q_{n}=C e^{-\eta n}$, where $C$ is a non-zero constant, into the linear part of equation (16), that is

$$
\varepsilon\left(Q_{n+1}-2 Q_{n}+Q_{n-1}\right)-Q_{n}=0,
$$

which yields

$$
-\varepsilon^{2}\left(e^{-\eta}+e^{\eta}\right)=(2 c+1) \Rightarrow \eta=\operatorname{arccosh}\left(\frac{2 \varepsilon+1}{2 \varepsilon}\right) .
$$

Taylor expansion of $e^{\eta}$ at $\varepsilon \approx 0$ is given by

$$
e^{\eta}=\varepsilon-2 \varepsilon^{2}+5 \varepsilon^{3}-14 \varepsilon^{4}+\mathcal{O}\left(C^{5}\right) .
$$

Let us assume that parameters $A$ and $B$ can be written in the following form of expansion

$$
\begin{aligned}
& A=a_{0}+a_{1} \varepsilon+a_{2} \varepsilon^{2}+a_{3} \varepsilon^{3}+\mathcal{O}\left(\varepsilon^{4}\right), \\
& B=b_{0}+b_{1} \varepsilon+b_{2} \varepsilon^{2}+b_{3} \varepsilon^{3}+\mathcal{O}\left(\varepsilon^{4}\right),
\end{aligned}
$$

where $a_{i}$ and $b_{i}$ are coefficients that will be determined its values by substituting equations (30), (31) and (32) into equations (23) and (24), and then collect the resulting terms in successive powers of $\varepsilon$.

Next, substitution of ansatz (21) into equation (26) gives

$$
R_{n}(Q)=\left\{\begin{array}{c}
\varepsilon\left(A e^{\eta(n+2)}-2 A e^{\eta(n+1)}+A e^{\eta n}\right)-A e^{\eta(n+1)}+\frac{1}{2} \alpha A^{2}\left(e^{\eta(n+1)}\right)^{2} \\
\left(A e^{\eta(n+2)}+A e^{\eta n}\right)+(1-\alpha) A^{3}\left(e^{\eta(n+1)}\right)^{3}, \quad n \leq-2 \\
\varepsilon B-2 \varepsilon A+\varepsilon A e^{-\eta}-A+\frac{1}{2} \alpha A^{2} B+\frac{1}{2} \alpha A^{2} e^{-\eta}+A^{3}-A^{3} \alpha, \quad n=-1 \\
\varepsilon B-2 \varepsilon A+\varepsilon A e^{-\eta}-A+\frac{1}{2} \alpha A^{2} B+\frac{1}{2} \alpha A^{2} e^{-\eta}+A^{3}-A^{3} \alpha, \quad n=2 \\
\varepsilon\left(A e^{-\eta(n-1)}-2 A e^{-\eta(n-2)}+A e^{-\eta(n-3)}\right)-A e^{-\eta(n-2)}+\frac{1}{2} \alpha A^{2}\left(e^{-\eta(n-2)}\right)^{2} \\
\left(A e^{-\eta(n-1)}+A e^{-\eta(n-3)}\right)+(1-\alpha) A^{3}\left(e^{-\eta(n-2)}\right)^{3}, \quad n \geq 3
\end{array}\right.
$$


Upon substituting expansions (30), (31), (32) into equation (33), one can obtain for $\alpha \rightarrow 0$ as follows

$$
\begin{gathered}
R_{0,1}(Q)=0, \\
R_{-1, \pm 2}(Q)=\mathcal{O}\left(\varepsilon^{2}\right), \\
R_{n \leq-3}(Q)=\mathcal{O}\left(\varepsilon^{|n-3|}\right), \\
R_{n \geq 3}(Q)=\mathcal{O}\left(\varepsilon^{|n+2|}\right) .
\end{gathered}
$$

One can check that sequence $\left\{R_{i}(Q)\right\}_{i=1}^{\infty}$ converges to 0 . Therefore $\left|R_{1}\right|^{2}+\left|R_{2}\right|^{2}+\ldots$ is convergent or $\sum_{k=1}^{\infty}\left|R_{k}\right|^{2}<\infty$. This explains that $R_{i}(Q)$ is in $l_{2}$ space with norm $\|R(Q)\|_{l_{2}}=\sqrt{\left|R_{1}\right|^{2}+\left|R_{2}\right|^{2}+\ldots}$. Thus from (34), we have

$$
\|R(Q)\|_{l_{2}}=\mathcal{O}\left(\varepsilon^{2}\right) \text { as } \varepsilon, \alpha \rightarrow 0 .
$$

Moreover, let us suppose $S=\{0,1\}, e_{0}=(\ldots, 0,0,0,1,0,0,0, \ldots), e_{1}=$ $(\ldots, 0,0,0,1,0,0, \ldots), \sigma_{0}=C$, and $\sigma_{1}=C$ for $C>0$. For the case of intersite discrete soliton, it can be shown that the variational solution $Q_{*}$ expressed by ansatz (21) satisfies the relationship

$$
\lim _{\varepsilon, \alpha \rightarrow 0}\left\|Q_{*}-\left(\sigma_{0} e_{0}+\sigma_{1} e_{1}\right)\right\|_{l_{2}}=0 .
$$

Based on Chong et al. [10], there is $\varepsilon_{0}, K>0$ and the unique solution $Q$ of stationary AL-cubic DNLS equation (16) with $\varepsilon \in\left(0, \varepsilon_{0}\right)$ such that

$$
\left\|Q-Q_{*}\right\|_{l_{2}} \leq K \varepsilon^{2}
$$

Since the exact solution is unknown, in practice the quantity of $\left\|Q-Q_{*}\right\|_{l_{2}}$ can be replaced by

$$
\text { error }=\left\|Q_{\text {num }}-Q_{*}\right\|,
$$

where $Q_{\text {num }}$ is the numerical solution. As an illustration, suppose the validation of the VA results will be checked for parameter value $\alpha=0.001$. Plot of error (38) in varied $\varepsilon$ for such $\alpha$ is given in Fig. 2.

From Fig. 2, it can be seen that the error gets bigger as $\varepsilon$ increases. To find out the function of error curve, we perform the best power fit, which gives $f(\varepsilon)=1.2997 \varepsilon^{2.0032}$. Note this result satisfies equation (27) in Proposition 1. This confirms that the VA solution for intersite soliton using ansatz (21) is valid. 


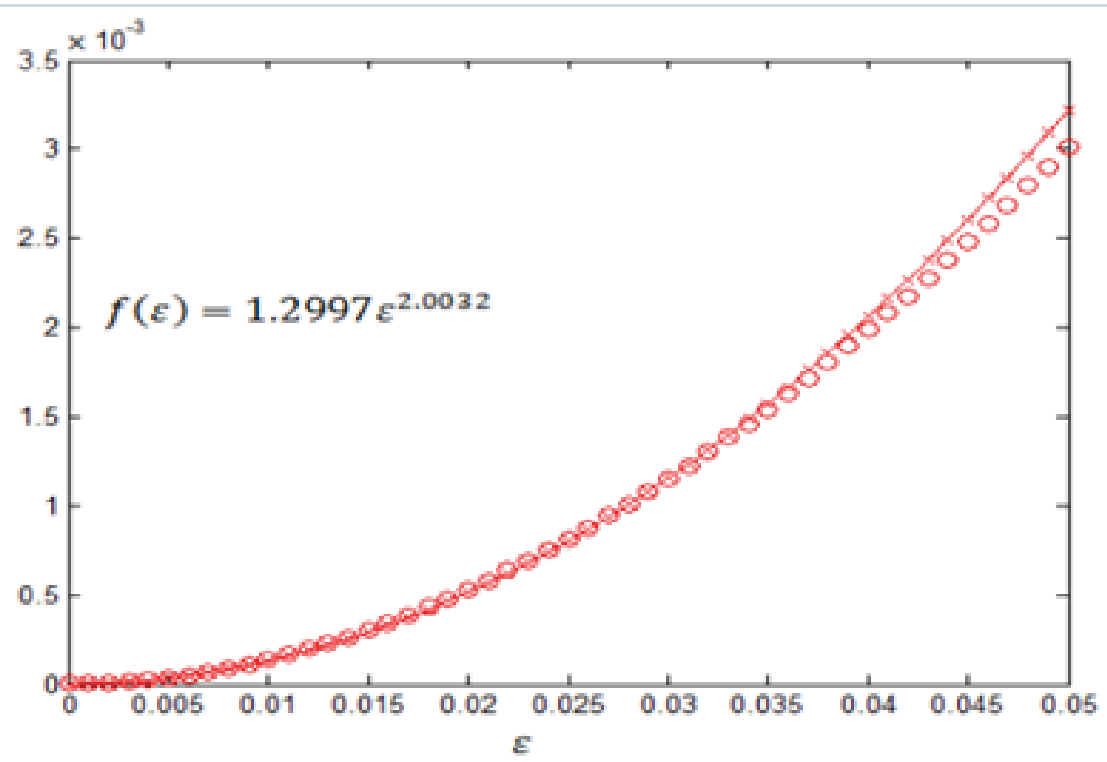

Figure 2: The error between variational and numerical solutions in varied $\varepsilon$ for $\alpha=0.001$.

\section{Conclusion}

Variational approximation (VA) developed for stationary intersite soliton in the ALcubic DNLS equation gives very good results for small coupling constant and small interpolation parameter. Following reference [10], we also show that the obtained VA solutions are valid.

\section{References}

[1] Eisenberg H.S., Silberberg Y., Morandotti R., Boyd A. R., and Aitchison J. S. (1998). Phys.Rev. Lett. 81: 3383.

[2] Kevrekidis P. G. (2009). Discrete Nonlinear Schrödinger Equation: Mathematical Analysis,Numerical Computations and Physical Perspectives. Berlin: Springer.

[3] Drazin, P.G. (1989). Soliton: An Introduction. Cambridge University Press, Cambridge.

[4] Tagg, C. (2005). Soliton Theory in Optical Communications. In Annual Review of Broadband Communications. Chicago: International Engineering Consortium.

[5] Ablowitz, M.J and Ladik, J.F. (1976). Nonlinear Differential-Difference Equations. J. Math. Phys. 16: 598.

[6] Goldstein, H., Poole C., and Safko J. (2002). Classical Mechanics. San FranciscoCalif: Addison Wesley.

[7] Aceves, et. al. (1996). Discrete Self-Trapping, Solitons Interactions, and Beam Steering in Nonlinear Waveguide Arrays. Phys. Rev. E. 53: 1172. 
[8] Cuevas, J., James, G., Kevrekidis, P.G., Malomed, B.A., and SanchezRey, B. (2008). Approximation of Solitons in the Discrete NLS Equation. J. Nonlinear Math, Phys. 15: 124.

[9] Kaup, D.J. (2005). Variational Solutions for the Discrete Nonlinear Schrödinger Equation. Math. Comput. Simulat. 69: 322.

[10] Chong, C., Pelinovsky, D. E., and Schneider, G. (2011). On the validity of the variational approximation in discrete nonlinear Schrodinger equation. Physica D 241: 115.

[11] Dawes, J.H.P and H. Susanto. (2013). Variational Approximation and the Use of Collective Coordinates. Phys. Rev. E. 87: 06320231. 